Pacific Journal of Mathematic 


\section{THE PRODUCT FORMULA FOR THE THIRD OBSTRUCTION}

ROBERT E. MOSHER

Let $\xi$ be an $S O(n)$-bundle with $n>3$; let $p$ : $E \rightarrow B$ be the projection in the associated $(n-1)$-sphere bundle. In this note we express the third obstruction to a cross-section of $p$ as a tertiary characteristic class and prove a product formula for the behavior of this class under Whitney sum.

The first obstruction is the Euler class $\chi(\xi) \in H^{n}(B ; Z) . \quad \chi$ is a primary characteristic class and satisfies $\chi=j^{*}(U)$, where $j: B \rightarrow T$ is the inclusion into the Thom space and $U \in H^{n}(T ; Z)$ is the Thom class. Whenever $\chi(\xi)=0$, a secondary characteristic class

$$
\alpha(\xi) \in H^{n+1}\left(B ; Z_{2}\right) /\left(S q^{2}+w_{2} \smile\right) H^{n-1}(B ; Z)
$$

is defined. $\alpha$ is the second obstruction and satisfies

$$
\alpha=\left(S q^{2}+w_{2} \smile\right)_{j}(U) \text {. }
$$

Thus $\alpha$ is obtained by applying a twisted functional primary operation to $U$. The third obstruction $\gamma(\xi)$, defined whenever $\alpha(\xi) \equiv 0$, will be expressed as the value $\Phi_{j}(U)$ of a certain twisted functional secondary operation.

It is immediately plausible to consider as $(n+1)$-ary characteristic classes the values of certain functional twisted $n$-ary operations on $U$, defined when appropriate $n$-ary characteristic classes vanish. We hope to deal with such classes systematically in a future paper, but the treatment is expected to be more complicated technically; hence $\gamma(\xi)$ is presented here as an illustrative example in a straightforward setting.

The paper is organized as follows. Section 2 is a statement of results, while in $\S 3$ we define $\gamma(\xi)$. The Peterson-Stein formula and the proof of $(2.2)$ appears in $\S 4$; the product formula is obtained in $\S 5$. We conclude in $\S 6$ with an example.

Throughout the paper all cohomology is taken with $Z_{2}$ as coefficients unless otherwise indicated.

2. Statement of results. Suppose $\xi$ is an $S O(n)$-bundle with $n>3$ and suppose $\chi(\xi)=0$. Let

$$
\alpha(\xi) \in H^{n+1}(B) /\left(S q^{2}+w_{2} \smile\right) H^{n-1}(B ; Z)
$$

be the secondary characteristic class given by $\alpha(\xi)=\left(S q^{2}+w_{2} \smile\right)_{j}(U)$ 
$[5,6,7,9]$. By [9], $\alpha(\xi)$ is the second obstruction to a cross-section in the associated sphere bundle.

Suppose now $\alpha(\xi) \equiv 0$. Then in $\S 3$ is defined a tertiary characteristic class $\gamma(\xi) \in H^{n+2}(B)$ modulo an indeterminacy $Q$, given in (3.6). $\gamma$ is natural in the following sense.

Proposition 2.1. $f: \xi^{\prime} \rightarrow \xi$ be a map of $S O(n)$-bundles. Suppose $\gamma(\xi)$ is defined. Then $\gamma\left(\xi^{\prime}\right) \equiv f^{*}(\gamma(\xi)) \bmod Q\left(\xi^{\prime}\right)$.

In $\S 4$ we establish the following.

Proposition 2.2. $\gamma(\xi)$ is the third obstruction to a cross-section of $p$.

For product farmulas we now assume $\xi$ and $\xi^{\prime}$ are $S O(n)$ and $S O\left(n^{\prime}\right)$ bundles over $B$ and $B^{\prime}$ respectively such that $\alpha(\xi)$ and $\alpha\left(\xi^{\prime}\right)$ are defined. Let $\xi \oplus \xi^{\prime}$ be the external Whitney sum over $B \times B^{\prime}$. By the Whitney formula for secondary characteristic classes [9], $\alpha\left(\xi \oplus \xi^{\prime}\right) \equiv 0$ and thus $\gamma\left(\xi \oplus \xi^{\prime}\right)$ is defined. In $\S 5$ we prove the following.

Proposition 2.3. $\gamma\left(\xi \oplus \xi^{\prime}\right) \equiv \alpha(\xi) \otimes \alpha\left(\xi^{\prime}\right)$ modulo the total indeterminacy.

Taking $B=B^{\prime}$ and writing $\xi+\xi^{\prime}$ for the internal Whitney sum, we obtain the following corollary to (2.1) and (2.3).

Proposition 2.4. $\gamma\left(\xi+\xi^{\prime}\right) \equiv \alpha(\xi) \smile \alpha\left(\xi^{\prime}\right)$ modulo the total indeterminacy.

3. Definition of $\gamma(\xi)$. Let $A$ be the $\bmod 2$ Steenrod algebra. In the semi-tensor product $H^{*}(B S O) \odot A$ [3] we have, in the terminology of [11], the relation

$$
\left(1 \otimes S q^{2}+w_{2} \otimes 1\right)\left(1 \otimes S q^{2}+w_{2} \otimes 1\right)=0
$$

over $Z$. Let $\beta=1 \otimes S q^{2}+w_{2} \otimes 1$. According to [4] and [11], (3.1) defines for each $n$ sufficiently large $(n>2$ suffices in this case) a twisted secondary operation $\Phi^{(n)} . \quad \Phi^{(n)}$ is defined on an $n$-dimensional integral cohomology class $x$ of a space $X$, where $\beta x=0$ and $H^{*}(B S O) \times A$ acts on the cohomology of $X$ via a vector bundle. The indeterminacy of $\Phi^{(n)}(X)$ is the subgroup $\beta H^{n+1}(X)$ of $H^{n+3}(X)$. While $\Phi^{(n)}$ is not uniquely determined by (3.1), computation in the universal example verifies the following for $n>2$.

Proposition 3.2. For each $n$, there exist precisely two distinct 
operations $\Phi_{1}^{(n)}$ and $\Phi_{2}^{(n)}$ associated with (3.1); these operations are related by $\Phi_{1}^{(n)}(x)+\Phi_{2}^{(n)}(x)=S q^{3} x=w_{3} \smile x$.

Let $U_{n}$ be the Thom class of the universal $S O(n)$-bundle $\gamma_{n}$. Another calculation checks the following.

Proposition 3.3. For each $n$, there is a unique choice of $\Phi^{(n)}$ such that $\Phi^{(n)}\left(U_{n}\right)=0$.

We now assume that $\Phi^{(n)}$ are so chosen and further note that $\Phi^{(n)}$ so chosen are compatible with coboundary, as is verified by consideration of the natural map $T\left(\gamma_{n-1}+1\right) \rightarrow T\left(\gamma_{n}\right)$ of Thom spaces.

Suppose now the $S O(n)$-bundle $\xi$ satisfies $\chi(\xi)=0$ and $\alpha(\xi) \equiv 0$. Then $U$ satisfies $j^{*}(U)=0, \beta(U)=0, \beta_{j}(U) \equiv 0$, and $\Phi(U)=0$ with zero indeterminacy. Under these circumstances one defines $\Phi_{j}(U)$ by the analogue for twisted operations of Peterson's generalization [8] of Steenrod's basic method [10], detailed below; one then defines $\gamma(\xi)$ as follows.

DeFinition 3.4. $\gamma(\xi)=\Phi_{j}(U)$.

To define $\Phi_{j}(U)$, following Massey [2], consider the cohomology sequence of the pair $(B, E)$ where $B$ replaces the mapping cylinder of $p$. Since $\chi(\xi)=j^{*}(U)=0$, we may choose $a \in H^{n-1}(E ; Z)$ such that $\delta^{*}(a)=U$. Since $\alpha(\xi) \equiv 0, a$ may be further assumed to satisfy $\beta(a)=0$. Then $\Phi(a)$ is defined and satisfies

$$
\delta^{*} \Phi(a)=\Phi\left(\delta^{*}(a)\right)=\Phi(U)=0 .
$$

DeFinition 3.5. $p^{*}\left(\Phi_{j}(U)\right)=\bigcup \Phi(a)$ as $a$ ranges over elements $a \in H^{n-1}(E ; Z)$ such that $\delta^{*}(a)=U$ and $\left(S q^{2}+w_{2} \smile\right)(a)=0$.

Proposition 3.6. The indeterminacy $Q$ of $\gamma(\xi)$ is given by

$$
Q=\{\Phi(b)+\beta(c)\},
$$

where $b \in H^{n-1}(B ; Z)$ such that $\Phi(b)$ is defined and $c \in H^{n}(B)$.

(3.6) and (2.1) are now evident.

4. The Peterson-Stein formula and the proof of (2.2). Twisted secondary operations satisfy the usual Peterson-Stein formulas. Stated as (4.1), for simplicity in terms of absolute cohomology classes, is the one to be used. 
Proposition 4.1. Let $f: Y \rightarrow X$ be a map compatible with the given structures of $Y$ and $X$ as spaces obtained from vector bundles. Let $x \in H^{n}(X ; Z)$ satisfy $\beta\left(f^{*}(x)=0\right.$. Then

$$
\Phi\left(f^{*}(x)\right) \equiv \beta_{f} \beta(x) \in H^{n+3}(Y) \bmod \beta H^{n+1}(Y)+f^{*} H^{n+3}(X) .
$$

The proof of (4.1) is postponed to the end of this section. The functional operation $\beta_{f}$ appearing in (4.1) is defined by the generalization of Steenrod's method as given in [7].

We now turn to the proof of (2.2). Consider the portion of the Moore-Postinkov tower for the associated sphere bundle to the universal $S O(n)$-bundle $\gamma_{n}$ displayed in (4.2).

Diagram 4.2.

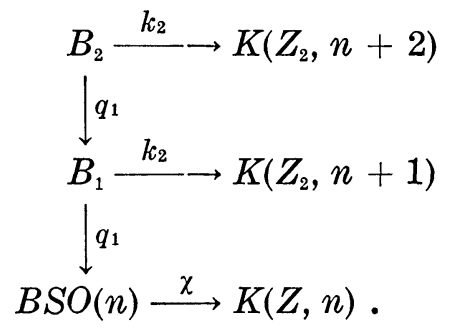

Let $\xi_{1}=q_{1}^{*}\left(\gamma_{n}\right)$ and $\xi_{2}=q_{2}^{*}\left(\xi_{1}\right)$. It then suffices to show $k_{2} \in \gamma\left(\xi_{2}\right)$. By [9] $k_{1} \in \alpha\left(\xi_{1}\right)$, while, by [1], $k_{2} \in \beta_{q_{2}}\left(k_{1}\right)$.

Consider now (4.3), induced by the bundle map $q_{2}: \xi_{2} \rightarrow \xi_{1}$.

Diagram 4.3.

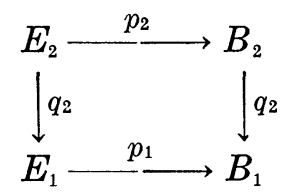

Since $k_{1} \in \alpha\left(\xi_{1}\right)$, we may write $p_{1}^{*}\left(k_{1}\right)=\beta\left(a_{1}\right)$ for an appropriate $a_{1} \in H^{n-1}\left(E_{1}\right)$ such that $\delta^{*}\left(a_{1}\right)=U\left(\xi_{1}\right)$. Let $a_{2}=q_{2}^{*}\left(a_{1}\right)$. Then $\left(p_{2}^{*}\right)^{-1} \Phi\left(a_{2}\right)$ represents $\gamma\left(\xi_{2}\right)$.

On the other hand, since $k_{2} \in \beta_{q_{2}}\left(k_{1}\right)$, by naturality

$$
p_{2}^{*}\left(k_{2}\right) \in \beta_{q_{2}}\left(p_{1}^{*}\left(k_{1}\right)\right)=\beta_{q_{2}} \beta\left(a_{1}\right) \text {. }
$$

The result follows by (4.1), which yields $\beta_{q_{2}} \beta\left(a_{1}\right) \equiv \Phi\left(a_{2}\right)$.

Proof of (4.1). For this proof we adopt the notations of [11]. Let $p: E, Y \rightarrow Y \times K, Y$ be the universal example for $\Phi$. Then a representative $\varphi$ of $\Phi\left(p^{*}\left(\ell_{n}\right)\right)$ is defined in [11] by means of a certain relative transgression sequence for $p$ by a formula $\varphi \in \mu^{-1} \alpha \tau^{-1} \beta\left(\iota_{n}\right)$. However, it is proved in [12] that this transgression sequence, in the range of dimensions considered, is equivalent to the cohomology sequence of the triple $(M, E, Y)$, where $M$ is the mapping cylinder of 
$p$. Let $j: Y \times K, Y \rightarrow M, E$ be the inclusion. Translating the definition of $\varphi$ to this sequence, we have $\varphi \in\left(\delta^{*}\right)^{-1} \beta\left(j^{*}\right)^{-1} \beta\left(\iota_{n}\right)$. But this last is precisely the definition of a representative of $\beta_{p} \beta\left(\iota_{n}\right)$. Thus (4.1) is valid in the universal example, and hence in general.

5. Proof of (2.3). We now consider bundles $\xi$ and $\xi^{\prime}$ such that $\alpha(\xi)$ and $\alpha\left(\xi^{\prime}\right)$ are defined; let $\xi^{\prime \prime}=\xi \oplus \xi^{\prime}$. Denote by $Z$ the mapping cylinder of $p$. The following is proved in [7].

Proposition 5.1. There is a natural homeomorphism of pairs $Z^{\prime \prime}$, $E^{\prime \prime} \rightarrow Z \times Z^{\prime}, E \times Z^{\prime} \smile Z \times E^{\prime}$ extending the identity of $B^{\prime \prime}=B \times B^{\prime}$ and inducing a natural homeomorphism $T^{\prime \prime} \rightarrow T^{\prime \prime} \wedge T^{\prime}$.

Now consider (5.2), in which the rows and the middle triangle are exact. The top row of (5.2) is obtained by splicing $\left(0 \rightarrow H^{*}(B) \rightarrow\right.$ $\left.H^{*}(E) \rightarrow H^{*}(T) \rightarrow 0\right) \otimes H^{*}\left(B^{\prime}\right)$ with $H^{*}(T) \otimes\left(0 \rightarrow H^{*}\left(B^{\prime}\right) \rightarrow H^{*}\left(E^{\prime}\right) \rightarrow\right.$ $H^{*}\left(T^{\prime}\right) \rightarrow 0$ ), while the triangle is the exact sequence of the pair $E^{\prime \prime}$, $E \times Z^{\prime}$.

Diagram 5.2.

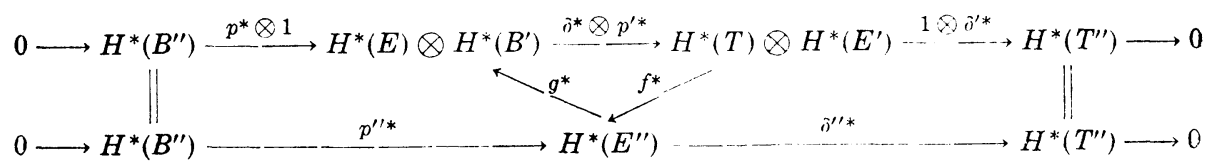

The proof of (2.3) is based on (5.2) as follows. Choose $a^{\prime} \in H^{n^{\prime}-1}\left(E^{\prime}\right)$ such that $\delta^{\prime *}\left(a^{\prime}\right)=U^{\prime}$. Let $a^{\prime \prime}=f^{*}\left(U \otimes a^{\prime}\right)$. Then $\delta^{\prime \prime *}\left(a^{\prime \prime}\right)=U^{\prime \prime}$. Further, $\left(S q^{2}+w_{2}^{\prime \prime} \smile\right)\left(a^{\prime \prime}\right)=0$, as calculation checks. Thus $\left(p^{\prime \prime *}\right)^{-1} \Phi\left(a^{\prime \prime}\right)$ is a representative of $\gamma\left(\xi+\xi^{\prime}\right)$.

On the other hand, $\Phi\left(a^{\prime \prime}\right)=\Phi\left(f^{*}\left(U \otimes a^{\prime}\right)\right)$ may be evaluated by (4.1). Computing, using the $\mathrm{Wu}$ formula [9] $\left(S q^{2}+w_{2} \smile\right)(a)=0$ and denoting by $a$ any class in $H^{n-1}(E ; Z)$ such that $\delta^{*}(a)=U$, we have the following, in which $\alpha(\alpha)$ is the representative of $\alpha$ determined by $\alpha$.

$$
\begin{aligned}
\left(p^{\prime *}\right)^{-1} \Phi\left(a^{\prime \prime}\right) & =\left(p^{\prime * *}\right)^{-1} \Phi\left(f^{*}\left(U \otimes \alpha^{\prime}\right)\right) \\
& =\left(p^{\prime \prime *}\right)^{-1} \beta_{f}^{\prime \prime} \beta^{\prime \prime}\left(U \otimes \alpha^{\prime}\right) \\
& =\left(p^{\prime \prime *}\right)^{-1} \beta_{f}^{\prime \prime}\left[U \otimes \beta^{\prime}\left(a^{\prime}\right)\right] \\
& =\left(p^{\prime \prime *}\right)^{-1}\left(g^{*}\right)^{-1} \beta^{\prime \prime}\left[\alpha \otimes \alpha^{\prime}\left(\alpha^{\prime}\right)\right] \\
& =\left(p^{*} \otimes 1\right)^{-1}\left[\beta(a) \otimes \alpha^{\prime}\left(\alpha^{\prime}\right)\right] \\
& =\alpha(\alpha) \otimes \alpha^{\prime}\left(\alpha^{\prime}\right)
\end{aligned}
$$

modulo indeterminacies.

This completes the proof of (2.3) and in fact of the following sharpening. 
COROLlaRY 5.3. Under the hypotheses of (2.3), let $\alpha(\alpha)$ and $\alpha^{\prime}\left(a^{\prime}\right)$ be representatives of $\alpha(\xi)$ and $\alpha\left(\xi^{\prime}\right)$ respectively. Then $\alpha(\alpha) \otimes \alpha^{\prime}\left(\alpha^{\prime}\right)$ is a representative of $\gamma\left(\xi \oplus \xi^{\prime}\right)$.

6. An example. Let $\xi+1$ be the tangent bundle of $S^{4 q+1}$ and $\xi^{\prime}+1$ the tangent bundle of $S^{4 q^{\prime}+1}$ for $q, q^{\prime} \geqq 1$. By [9], $\alpha(\xi) \neq 0 \bmod 0$ in $H^{4 q+1}\left(S^{4 q+1}\right)$ and similarly for $\xi^{\prime}$. It follows by (2.3) that $\gamma\left(\xi \oplus \xi^{\prime}\right)$ is nonzero in $H^{4 q+4 q^{\prime}+2}\left(S^{4 q+1} \times S^{4 q^{\prime}+1}\right)$; the indeterminacy again vanishes. Thus $\xi \oplus \xi^{\prime}$ has no nonvanishing section.

This result can be obtained without the use of twisted operations, for the Whitney classes here vanish. That $\alpha(\xi) \neq 0$ reflects that $S q^{2} a$ generates $p^{*} H^{4 q+1}\left(S^{4 q+1}\right)$ in $H^{4 q+1}(E)$, while $\gamma\left(\xi+\xi^{\prime}\right) \neq 0$ reflects that $\Phi_{1,1}\left(a^{\prime \prime}\right)$ generates $p^{\prime \prime *} H^{4 q+4 q^{\prime}+2}\left(S^{4 q+1} \times S^{4 q^{\prime}+1}\right)$ in $H^{4 q+4 q^{\prime}+2}\left(E^{\prime \prime}\right)$, where $\Phi_{1,1}$ is the ordinary secondary operation associated with the Adem relation $S q^{2} S q^{2}=0$, valid on integer classes.

\section{REFERENCES}

1. M. Mahowald, On obstruction theory in orientable fiber bundles, Trans. Amer. Math. Soc. 110 (1964), 315-349.

2. W. S. Massey, On the cohomology ring of a sphere bundle, J. Math. Mech. 7 (1958), 265-290.

3. W. S. Massey and F. P. Peterson, The cohomology structure of certain fibre spaces, I, Topology 4 (1964), 47-66.

4. J. F. McClendon, Higher order twisted cohomology operations, Ph. D. thesis, University of California, Berkeley, 1966.

5. J. P. Meyer, Functional cohomology operations and relations, Amer. J. Math. 97 (1965), 649-683.

6. R. E. Mosher, Functional cohomology operations and secondary characteristic classes, Ph. D. thesis, M.I.T., 1962.

7. - Secondary characteristic classes for k-special bundles, Trans. Amer. Math. Soc. 131 (1968), 333-344.

8. F. P. Peterson, Functional cohomology operations, Trans. Amer. Math. Soc. 86 (1957), 197-211.

9. F. P. Peterson and N. Stein, Secondary characteristic classes, Ann. of Math. (2) 76, (1962), 510-523.

10. N. E. Steenrod, Cohomology invariants of mappings, Ann. of Math. (2) 50 (1949), 954-988.

11. E. Thomas, Postnikov invariants and higher order cohomology operations, Ann. of Math. (2) 85 (1967), 184-217.

12. — An exact sequence for principal fibrations, (to appear)

Received January 4, 1968. Partially supported by NSF grant GP-8955.

California State College at Long Beach 


\section{PACIFIC JOURNAL OF MATHEMATICS}

\section{EDITORS}

\section{H. ROYDEN}

Stanford University

Stanford, California

\section{J. Dugundu}

Department of Mathematics University of Southern California Los Angeles, California 90007

RICHARD ARENS

University of California Los Angeles, California 90024

ASSOCIATE EDITORS
E. F. Beckenbach
B. H. NEUMANN
F. WoLF
K. YosidA

\section{SUPPORTING INSTITUTIONS}

\author{
UNIVERSITY OF BRITISH COLUMBIA \\ CALIFORNIA INSTITUTE OF TECHNOLOGY \\ UNIVERSITY OF CALIFORNIA \\ MONTANA STATE UNIVERSITY \\ UNIVERSITY OF NEVADA \\ NEW MEXICO STATE UNIVERSITY \\ OREGON STATE UNIVERSITY \\ UNIVERSITY OF OREGON \\ OSAKA UNIVERSITY \\ UNIVERSITY OF SOUTHERN CALIFORNIA
}

STANFORD UNIVERSITY

UNIVERSITY OF TOKYO

UNIVERSITY OF UTAH

WASHINGTON STATE UNIVERSITY

UNIVERSITY OF WASHINGTON

AMERICAN MATHEMATICAL SOCIETY
CHEVRON RESEARCH CORPORATION
TRW SYSTEMS

AMERICAN MATHEMATICAL SOCIETY

TRW SYSTEMS

NAVAL WEAPONS CENTER

Mathematical papers intended for publication in the Pacific Journal of Mathematics should be in typed form or offset-reproduced, double spaced with large margins. Underline Greek letters in red, German in green, and script in blue. The first paragraph or two must be capable of being used separately as a synopsis of the entire paper. It should not contain references to the bibliography. Manuscripts, in duplicate if possible, may be sent to any one of the four editors. All other communications to the editors should be addressed to the managing editor, Richard Arens, University of California, Los Angeles, California 90024.

Each author of each article receives 50 reprints free of charge; additional copies may be obtained at cost in multiples of 50 .

The Pacific Journal of Mathematics is published monthly. Effective with Volume 16 the price per volume (3 numbers) is $\$ 8.00$; single issues, $\$ 3.00$. Special price for current issues to individual faculty members of supporting institutions and to individual members of the American Mathematical Society: $\$ 4.00$ per volume; single issues $\$ 1.50$. Back numbers are available.

Subscriptions, orders for back numbers, and changes of address should be sent to Pacific Journal of Mathematics, 103 Highland Boulevard, Berkeley 8, California.

Printed at Kokusai Bunken Insatsusha (International Academic Printing Co., Ltd.), 7-17, Fujimi 2-chome, Chiyoda-ku, Tokyo, Japan.

PUBLISHED BY PACIFIC JOURNAL OF MATHEMATICS, A NON-PROFIT CORPORATION

The Supporting Institutions listed above contribute to the cost of publication of this Journal, but they are not owners of publishers and have no responsibility for its content or policies. 


\section{Pacific Journal of Mathematics \\ Vol. 27, No. 3}

March, 1968

Charles A. Akemann, Invariant subspaces of $C(G) \ldots \ldots \ldots \ldots \ldots \ldots . \ldots 41$

Dan Amir and Zvi Ziegler, Generalized convexity cones and their duals ... . 425

Raymond Balbes, On ( $J, M, \mathrm{~m})$-extensions of order sums of distributive

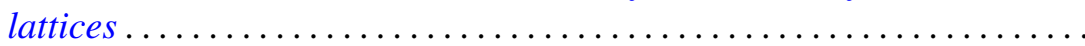

Jan-Erik Björk, Extensions of the maximal ideal space of a function algebra ........................................ 453

Frank Castagna, Sums of automorphisms of a primary abelian group ...... 463

Theodore Seio Chihara, On determinate Hamburger moment problems ..... .

Zeev Ditzian, Convolution transforms whose inversion function has complex

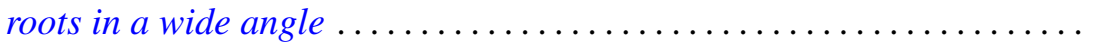

Myron Goldstein, On a paper of Rao .

Velmer B. Headley and Charles Andrew Swanson, Oscillation criteria for

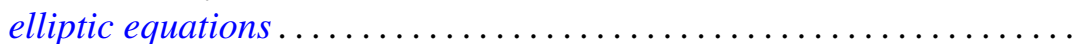

John Willard Heidel, Qualitative behavior of solutions of a third order nonlinear differential equation............................

Alan Carleton Hindmarsh, Pick's conditions and analyticity.............

Bruce Ansgar Jensen and Donald Wright Miller, Commutative semigroups

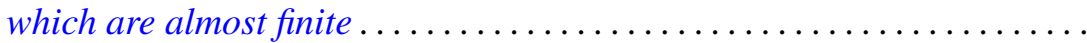

Lynn Clifford Kurtz and Don Harrell Tucker, An extended form of the

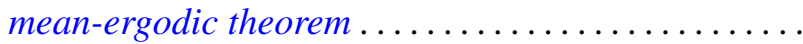

S. P. Lloyd, Feller boundary induced by a transition operator ...

Henry B. Mann, Josephine Mitchell and Lowell Schoenfeld, A new proof of the maximum principle for doubly-harmonic functions ...

Robert Einsohn Mosher, The product formula for the third obstruction ..

Sam Bernard Nadler, Jr., Sequences of contractions and fixed points ...

Eric Albert Nordgren, Invariant subspaces of a direct sum of weighted shifts...

Fred Richman, Thin abelian p-groups ...

Jordan Tobias Rosenbaum, Simultaneous interpolation in $\mathrm{H}_{2}$. II ...

Charles Thomas Scarborough, Minimal Urysohn spaces .

Malcolm Jay Sherman, Disjoint invariant subspaces..... .

Joel John Westman, Harmonic analysis on groupoids....

621

William Jennings Wickless, Quasi-isomorphism and TFM

Minoru Hasegawa, Correction to "On the convergence of resolvents of operators" 